\title{
Dissociating the effects of semantic grouping and rehearsal strategies on event-related brain potentials
}

Citation for published version (APA):

Schleepen, T. M. J., Markus, C. R., \& Jonkman, L. M. (2014). Dissociating the effects of semantic grouping and rehearsal strategies on event-related brain potentials. International Journal of Psychophysiology, 94(3), 319-328. https://doi.org/10.1016/j.ijpsycho.2014.09.007

Document status and date:

Published: 01/12/2014

DOI:

10.1016/j.ijpsycho.2014.09.007

Document Version:

Publisher's PDF, also known as Version of record

Document license:

Taverne

Please check the document version of this publication:

- A submitted manuscript is the version of the article upon submission and before peer-review. There can be important differences between the submitted version and the official published version of record.

People interested in the research are advised to contact the author for the final version of the publication, or visit the DOI to the publisher's website.

- The final author version and the galley proof are versions of the publication after peer review.

- The final published version features the final layout of the paper including the volume, issue and page numbers.

Link to publication

\footnotetext{
General rights rights.

- You may freely distribute the URL identifying the publication in the public portal. please follow below link for the End User Agreement:

www.umlib.nl/taverne-license

Take down policy

If you believe that this document breaches copyright please contact us at:

repository@maastrichtuniversity.nl

providing details and we will investigate your claim.
}

Copyright and moral rights for the publications made accessible in the public portal are retained by the authors and/or other copyright owners and it is a condition of accessing publications that users recognise and abide by the legal requirements associated with these

- Users may download and print one copy of any publication from the public portal for the purpose of private study or research.

- You may not further distribute the material or use it for any profit-making activity or commercial gain

If the publication is distributed under the terms of Article $25 \mathrm{fa}$ of the Dutch Copyright Act, indicated by the "Taverne" license above, 


\title{
Dissociating the effects of semantic grouping and rehearsal strategies on event-related brain potentials
}

\author{
T.M.J. Schleepen ${ }^{\text {a,* }}$, C.R. Markus ${ }^{\text {b }}$, L.M. Jonkman ${ }^{\text {a }}$ \\ a Department of Cognitive Neuroscience, Faculty of Psychology and Neuroscience, Maastricht University, The Netherlands \\ ${ }^{\mathrm{b}}$ Department of Neuropsychology and Psychopharmacology, Faculty of Psychology and Neuroscience, Maastricht University, The Netherlands
}

\section{A R T I C L E I N F O}

\section{Article history:}

Received 7 April 2014

Received in revised form 25 August 2014

Accepted 11 September 2014

Available online 18 September 2014

\section{Keywords:}

Elaborative memory strategies

Semantic grouping

Rehearsal

ERPs

Encoding

Maintenance

\begin{abstract}
A B S T R A C T
The application of elaborative encoding strategies during learning, such as grouping items on similar semantic categories, increases the likelihood of later recall. Previous studies have suggested that stimuli that encourage semantic grouping strategies had modulating effects on specific ERP components. However, these studies did not differentiate between ERP activation patterns evoked by elaborative working memory strategies like semantic grouping and more simple strategies like rote rehearsal. Identification of neurocognitive correlates underlying successful use of elaborative strategies is important to understand better why certain populations, like children or elderly people, have problems applying such strategies. To compare ERP activation during the application of elaborative versus more simple strategies subjects had to encode either four semantically related or unrelated pictures by respectively applying a semantic category grouping or a simple rehearsal strategy. Another goal was to investigate if maintenance of semantically grouped vs. ungrouped pictures modulated ERP-slow waves differently. At the behavioral level there was only a semantic grouping benefit in terms of faster responding on correct rejections (i.e. when the memory probe stimulus was not part of the memory set). At the neural level, during encoding semantic grouping only had a modest specific modulatory effect on a fronto-central Late Positive Component (LPC), emerging around 650 ms. Other ERP components (i.e. P200, N400 and a second Late Positive Component) that had been earlier related to semantic grouping encoding processes now showed stronger modulation by rehearsal than by semantic grouping. During maintenance semantic grouping had specific modulatory effects on left and right frontal slow wave activity. These results stress the importance of careful control of strategy use when investigating the neural correlates of elaborative encoding.
\end{abstract}

(c) 2014 Elsevier B.V. All rights reserved.

\section{Introduction}

Learning enables us to acquire the skills and knowledge to be successful in school and in life more generally. Memory strategies are tools that help us to structure information in such a way that it can be learned (and retrieved from memory) more easily, e.g. learning the sequence $\mathrm{u}-\mathrm{s}-\mathrm{a}-\mathrm{f}-\mathrm{b}-\mathrm{i}$ as meaningful chunks (i.e. USA and FBI). Especially when the material we need to study exceeds our memory span memory strategies can reduce memory load by leading to storage of information in a more organized way. Craik and Lockhart (1972) stressed that especially elaborative encoding (as opposed to shallow encoding as for example on perceptual features) led to improved memory due to the forming of more robust memory traces. Elaboration is the process of making information more meaningful by forming relations between its different parts (by for instance making images, chunks, semantic

\footnotetext{
* Corresponding author at: Department of Cognitive Neuroscience, Faculty of Psychology and Neuroscience, Maastricht University, P.O. Box 616, 6200 MD Maastricht, The Netherlands. Tel.: + 31433881522.

E-mail address: Tamara.schleepen@maastrichtuniversity.nl (T.M.J. Schleepen).
}

groups), often based on information/knowledge stored in long-term memory (LTM).

Whereas elaborative encoding strategies like semantic grouping have been shown to aid memory and learning more than simple strategies like rote rehearsal, their spontaneous use has been shown to be limited in certain populations like younger children and the elderly or those with attention disorders (Bjorklund and de Marchena, 1984; Wegesin et al., 2000; Egeland et al., 2010). Since such reduced elaborative strategy application has been linked to impaired memory performance it is important to identify the cognitive and neurobiological factors underlying (or limiting) its use. Behavioral studies suggest that one possible reason for the later (i.e. at a later age) application of elaborative strategies (e.g. semantic grouping) in childhood might be a limit in working memory capacity (Schneider et al., 2004; Schleepen and Jonkman, 2012). Functional MRI work in the elderly corroborates this by showing that learning to successfully use elaborative encoding strategies depends on working memory capacity and requires activation of different areas in the prefrontal cortex (PFC), including the dorsolateral PFC. In an fMRI study with young and older adults, Kirchhoff et al. (2012) for instance showed that older adults were only able to spontaneously initiate semantic encoding strategies after strategy 
training thereby increasing their memory recognition performance to the level of that of younger adults. Increased strategy use during during/after training went along with increased brain activity in prefrontal and left lateral temporal regions. Another study showed that also in young adults training by the use of a semantic grouping strategy during encoding led to increased recall and semantic clustering scores and cooccurred with increased activation in bilateral dorsolateral PFC and orbitofrontal cortex (Miotto et al, 2006). Moreover, several studies reported that prefrontal cortex activity was enhanced during the use of elaborative encoding strategies (vs. no strategy) even though such strategies facilitate memory performance and decrease WM-load (Bor et al., 2004; Bor et al., 2003; Bor and Owen, 2007; Kirchhoff and Buckner, 2006). Thus, these studies show that especially prefrontal cortex supports the acquisition and spontaneous use of elaborative encoding strategies.

Although the above imaging studies do provide us with important information about the brain areas involved in elaborative strategy application and learning, they do not inform us about the temporal course and duration of cognitive processes underlying such strategy use. Given the speed of neural processing during memory encoding, ERPs are needed to investigate this. Accordingly, the first aim of the present study is to dissociate spatio-temporal ERP correlates of elaborative (semantic grouping) versus simple (rehearsal) encoding strategies. Semantic grouping is an elaborative strategy that is used to improve verbal learning by reordering items into similar semantic categories (Mandler, 1967). It was chosen as the elaborative strategy in the present study since it was the focus of most prior developmental and ERPworking memory strategy work. Functional imaging studies have linked semantic grouping to left inferior and left dorsolateral PFC in adults, activity being larger during deliberate strategy application (Savage et al., 2001; Fletcher et al., 1998).

Although limited in number, several ERP studies also give some information about the ERP components reflecting different stages of cognitive processing involved in semantic grouping. In a study by Blanchet et al. (2007) EEG was recorded while subjects were presented with sequentially presented words in three encoding conditions that differed in the degree of required semantic grouping. While in the unrelated condition none of the words belonged to a similar semantic category, in both the spontaneous and guided conditions the words belonged to four different semantic categories. The two latter conditions differed from each other in that in the spontaneous condition participants were not informed about the semantic structure of the list and received no strategy instructions, while in the guided condition participants were given the names of the corresponding categories and were explicitly instructed to group the words on semantic category to aid later recall. Blanchet et al. reported several ERP components that were modulated by differences in semantic grouping demands. First, an increased P200 over predominantly fronto-central regions was observed that was the largest for guided vs. spontaneous vs. unrelated conditions. The P200 has been related to early stimulus encoding or detection processes (Picton and Hillyard, 1974) and more recently, to attentional processing during deep encoding (Mangels et al., 2001). The latter led Blanchet et al. to conclude that the largest P200 in the guided condition is caused by larger and faster attention allocation to the semantic features of the words in those conditions, since grouping categories were known beforehand. Second, a Late Positive Component (LPC) was found between 400 and $800 \mathrm{~ms}$ over centro-parietal regions. This component was the largest in both the guided and spontaneous conditions compared to the unrelated condition. The LPC was suggested to index voluntary associative processes involved in attempting to link words together that belong to similar semantic categories (e.g. semantic grouping). In general, the LPC has been elicited to stimuli across various modalities and has been related to processes as memory, attention and orienting (e.g. Courchesne et al., 1975; Hillyard and Picton, 1978). Finally, rightfrontal sustained slow wave activity was found that was increased in the spontaneous condition compared to both unrelated and guided conditions (600-1200 ms) and in the spontaneous condition vs. the unrelated condition (1200-1800 ms). Because the sustained right frontal slow wave was increased only in the spontaneous condition, Blanchet et al. associated this component with the degree of self-initiation involved in the application of the strategy. Besides the study of Blanchet et al. that directly studied ERP activity during the application of semantic grouping, other studies relevant for the present study are those that investigated which ERP components were modulated by retrieval of category-specific information from long-term memory (LTM). These studies reported modulations of a negative component around $400 \mathrm{~ms}$ above fronto-central, parietal and occipital electrodes and an occipitaltemporal/parietal positivity (LPC) around $550 \mathrm{~ms}$ when specific information about object categories had to be retrieved from semantic LTM (Kiefer, 2001, 2005). A similar negative component around $400 \mathrm{~ms}$ (called the N400) has been reported in the language processing-ERP literature, its amplitude being typically increased in response to semantic violations, e.g. "I like my coffee with sugar and shoes" (Kutas and Hillyard, 1980). Based on findings from semantic categorization studies that the N400 indexes activation of the semantic network (Kiefer, 2001, 2005), in this study we focus on the N400 as a measure most likely reflecting the retrieval of (category) information from semantic longterm memory during application of the semantic grouping strategy.

Although the above imaging and ERP studies give some insight in the neural correlates underlying semantic grouping during encoding, they did not directly compare neural correlates of semantic grouping with those evoked by (rote) rehearsal by giving explicit instructions for the application of both. In the above studies neural activity during semantic grouping was often contrasted with activity during trials in which unrelated material was presented and had to be encoded for later recall but in which no explicit strategy instructions were given. In such "no-instruction" trials (e.g. unrelated or related-spontaneous trials) it is not clear whether and which strategies subjects might have used. Because of the requirement of later recall it is however likely that subjects used simple rehearsal strategies (especially with sequential presentation of stimuli and low memory load), that might have also affected encoding ERPs. Thus it cannot be excluded that processes related to rehearsal may (partly) account for the increased activity reflected by the P2, LPC and late sustained potential in the ERP studies and prefrontal cortex in the fMRI studies in structured trials. This is an important issue since rehearsal has been shown to recruit partially overlapping brain networks (including left prefrontal cortex) as those underlying elaborative strategies (e.g. Smith and Jonides, 1999).

In the present study EEG was recorded while subjects encoded four simultaneously presented pictures of objects (S1) in two different strategy instruction conditions. In the semantic grouping condition these four pictures belonged to two semantic categories, and subjects were on each trial explicitly instructed to group these pictures on their corresponding semantic categories during encoding (they were not informed about the exact category names). In the rehearsal condition, the four pictures in S1 belonged to four different semantic categories (to prevent grouping on category), and here subjects received the explicit instruction to rehearse these pictures. In contrast to previous studies, this latter explicit rehearse instruction was given to investigate whether the ERPcomponents previously associated with semantic grouping are indeed relevant to semantic grouping or might also reflect involved rehearsal processes.

Besides comparing neural activation between different strategy instruction conditions during encoding, we also investigated potential differences in neural activations during maintenance of related (semantic grouping condition) or unrelated (rehearsal condition) pictures (i.e. when stimulus material was no longer visible). Therefore, following encoding, we asked subjects to maintain the four pictures in memory during a delay period until a probe occurred (S2). The processes of briefly retaining and updating/manipulating information in memory are referred to as working memory (WM) processes (Baddeley, 2000), typically giving rise to slow wave brain activity that can last up to several 
seconds (e.g. Mecklinger and Pfeifer, 1996; Ruchkin et al., 1992). Several studies have shown that the topography of such slow waves differs as a function of the type of material that is maintained in WM. Typically, (right) posterior slow waves were observed when spatial information was held in WM, while maintenance of phonological (verbal) material evoked negative slow waves over left frontal areas (Ruchkin et al., 1992; Bosch et al., 2001) and positive slow waves over bilateral posterior areas (Ruchkin et al., 1992). Other studies reported that the maintenance of object information in WM elicited right frontal slow waves (Mecklinger and Pfeifer, 1996; Mecklinger, 1998). In addition, task difficultly has also been reported to influence slow-wave amplitude; increased WM-load and more complex maintenance processes (e.g. updating versus simple rehearsal) generally lead to more negative slow wave amplitudes (Kiss et al., 2007; Ruchkin et al., 1992).

To conclude, based on prior research showing that deeper encoding of material leads to better memory performance (Craik and Lockhart, 1972), we expected increased accuracy and/or decreased reaction times in semantic grouping vs. rehearsal trials. Regarding ERPs during the encoding interval, we expected to see the first strategy effects on the P200, with higher amplitudes for semantic grouping vs. rehearsal trials based on prior findings that the P200 reflects allocation of attention that is higher when material is processed more deeply, like in semantic grouping (Blanchet et al., 2007). Furthermore, we expected to find increased N400 amplitude in semantic grouping vs. rehearsal trials, based on studies that reported effects of a semantic category manipulation on the $\mathrm{N} 400$ and suggested this is the stage at which subjects retrieve or have access to object category information in memory (Kiefer, 2001, 2005). The LPC, assumed to be related to the associative process of linking related items together (Blanchet et al., 2007), was also expected to be increased in semantic grouping vs. rehearsal trials. Finally, we do not expect to find effects on the right frontal sustained potential reported by Blanchet et al., since these effects were related to spontaneous initiation of the grouping strategy and our subjects received explicit strategy instructions in both types of trials.

On the basis of prior reports of increased activation in frontal brain areas when organized/grouped material is maintained in WM (Prabhakaran et al., 2000; Wendelken et al., 2008) and ERP studies showing that more complex maintenance processes give rise to larger amplitude ERP-slow waves (e.g. Kiss et al., 2007; Ruchkin et al., 1992), maintaining semantically grouped vs. ungrouped information in WM is hypothesized to elicit larger slow-wave ERP activity over frontal brain regions because the former is assumed to require elaborative (vs. simple rote) rehearsal. More specifically, because subjects were explicitly instructed to maintain the verbal codes in memory of the presented objects, we expected slow waves over left and right frontal areas which have been linked before to maintenance of phonological and object information (Mecklinger and Pfeifer, 1996; Mecklinger, 1998; Ruchkin et al., 1992; Bosch et al., 2001).

\section{Method}

\subsection{Subjects}

Nineteen adults, all university students, participated in this study (age range 18-25 years, mean age $=20.1$ years, 10 female). All subjects were free from neurological or psychiatric diseases and had normal or corrected-to-normal vision as verified by self-report. Informed consent was obtained from all participants, who were rewarded with course credit for their participation. The study was approved by a local ethical committee.

\subsection{Procedure}

The experimental session lasted approximately $3 \mathrm{~h}$ and took place in a dimly lit, sound attenuated room. Upon arrival, subjects first filled out the informed consent form, followed by attachment of the electrodes.
Next, participants were seated in front of a 17-inch VGA monitor with their eyes aligned to the center of the screen at a viewing distance of $75 \mathrm{~cm}$. Participants were instructed to minimize eye blinks and to refrain from making head or eye movements during execution of the experimental task. Before the experimental task was started, subjects performed a short practice task wherein they had to reach a predetermined performance criterion of $75 \%$ correct responses.

\subsection{Experimental task}

Participants performed a memory strategy task in which the sequence of events in a trial was as follows (see Fig. 1): A trial started with a $500 \mathrm{~ms}$ fixation cross, after which a $1000 \mathrm{~ms}$ cue (1st cue) was presented that contained the relevant strategy instruction for that trial (e.g. group, rehearse or view; see below). After a 500 ms fixation cross the stimulus display (S1; comprising four pictures of objects) was displayed for $4500 \mathrm{~ms}$ around a centrally presented fixation cross and was followed by a $5000 \mathrm{~ms}$ retention interval. A second cue (of $1000 \mathrm{~ms}$ duration) then informed the subjects of the decision they had to make when the following memory probe stimulus (S2) would appear (500 ms after the 2nd cue). The presentation duration of S2 was $1500 \mathrm{~ms}$ and responses given after S2 ended were coded as misses. Between S1 and S2 a centrally presented fixation cross was continuously present. There was a fixed intertrial interval of $1500 \mathrm{~ms}$.

There were three different strategy instruction cues defining the type of trial. The instruction "group the pictures" informed participants that they had to encode and hold S1 in memory during the S1-S2 interval by grouping the pictures on semantic category. The cue "rehearse the pictures" indicated that the S1 objects had to be named and that these picture names were to be rehearsed subvocally during the S1$\mathrm{S} 2$ interval. The instruction "look at the pictures" instructed subjects to only look at the pictures in S1 without the requirement to remember them for later recognition. The meaning of these instruction cues was elaborately explained to the subjects before the start of the practice session.

The S1 stimulus (the memory set) always consisted of four pictures of black and white drawing of objects that were randomly presented against a black background in an invisible $2 \times 2$ matrix measuring $13.5 \mathrm{~cm}\left(11.0^{\circ}\right)$ horizontally by $12\left(9.7^{\circ}\right) \mathrm{cm}$ vertically. The four pictures were drawn from a total set of 36 pictures each measuring $6.5\left(4.6^{\circ}\right)$ by $5.5 \mathrm{~cm}\left(5.4^{\circ}\right)$ selected from the published picture set by Cycowicz et al. (1997). These 36 object pictures belonged to the following 6 semantic categories: animals, fruits, clothes, body parts, furniture and vehicles and consisted of the 6 most familiar objects within each category (based on familiarity scores of 5-6 year-old children as published by Cycowicz et al.). In semantic grouping trials the four pictures in S1 always belonged to two different semantic categories ( 2 per category; related trials), whereas in rehearse trials all pictures were from different semantic categories (unrelated trials) to prevent subjects from using semantic grouping in spite of instructions. To be able to control for potential differences caused by these $\mathrm{S} 1$ stimulus differences there were two types of control trials with S1 pictures either belonging to two or four semantic categories (similar to in grouping and rehearse trials respectively). The pictures in the control task were also randomly presented in the $2 \times 2$ matrix. The memory set was kept deliberately below maximum capacity (i.e. above \pm 7 items; Miller, 1956) to be able to study strategy effects on ERPs without interfering effects of load. Maintaining WM-loads that exceed maximum capacity has been found to alter subject's strategies (Cowan, 2001) and to increase activation in the PFC (Rypma et al., 2002).

The second instruction cue that preceded the memory probe stimulus (S2) also differed between the two strategy conditions. In rehearse trials the cue preceding S2 was always the word "picture", instructing participants to decide whether the object stimulus presented in S2 was present in the preceding S1 memory set or not. In semantic grouping trials in addition to the picture cue a "category" cue was added that 
A

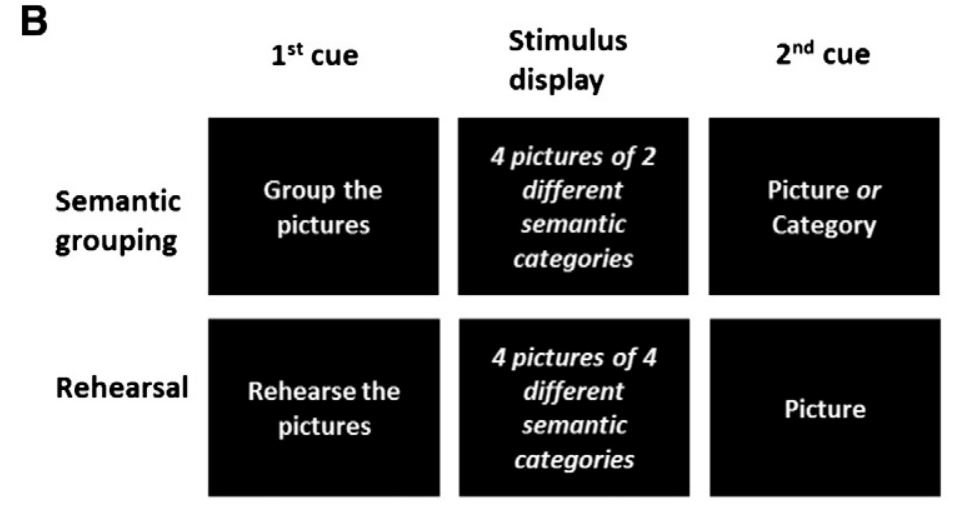

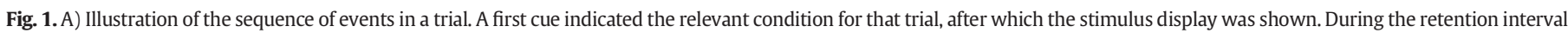

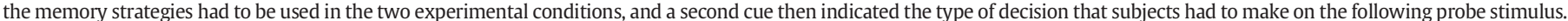
(B) Schematic illustration of the specific cues and configuration of object pictures in the stimulus display in the two experimental conditions.

required participants to decide whether the semantic category to which the $\mathrm{S} 2$ object belonged was similar or not to one of the semantic categories presented in the preceding S1 stimulus set. The extra category cue (presented on $50 \%$ of the grouping trials) was included to enhance the need for grouping on and maintaining the semantic categories and decrease the risk subjects just rehearsed the four individual S1 items. During a debriefing at the end of the experiment all participants indicated to have used the instructed strategies. Strategy adherence was also suggested by the behavioral data (see Results section).

Note that in trials that required rejection of the probe stimulus, the probe could either belong to a similar or a different category as shown in the S1-display; this was on each trial randomly determined. The control condition also had "picture" and a "category" instruction cues before S2 but here subjects had to respectively indicate whether two pictures presented at the left and right of the fixation cross were or were not the same or whether they belonged to the same semantic category or not. Since in the control condition S2 always consisted of two pictures, in the strategy conditions S2 also consisted of two (now the same) pictures to keep conditions perceptually similar (see Fig. 1A).

The total task consisted of 270 trials presented in 9 separate blocks of 30 trials each. The control and related-semantic grouping conditions each comprised 108 trials (54 S2-“category” probe trials and 54 S2-“picture" probe trials), while the unrelated-rehearsal condition consisted of 54 trials (only S2-picture trials). In each block, there were 12 control and semantic grouping trials ( 6 for picture and 6 for category probes) and 6 rehearse trials, presented completely random. Between blocks, participants were able to take a short break. Whereas semantic grouping and rehearsal conditions contained different numbers of trials, in the behavioral analyses only S2-picture probe trials were compared (comprising 54 trials in both strategy conditions). On all trials participants had to make a yes or no response by pressing the left- or right-hand response button using their index fingers, which was counterbalanced between participants. Participants were instructed to respond quickly, but never at the expense of accuracy. The task was programmed in the software package Presentation (Neurobehavioral Systems, Albany, CA; www.neurobs. com).

\subsection{Electrophysiological recording and analysis}

EEG activity was recorded from 60 scalp locations using $\mathrm{Ag} / \mathrm{AgCl}$ electrodes located placed in an elastic cap (EasyCap). Brain Vision Recorder software and the corresponding Brainamp amplifiers were 
used for signal acquisition (Brain Products $\mathrm{GmbH}$, version 1.10). One electrode in the EasyCap (FPz) was used as a ground, and the left mastoid functioned as the online reference for all electrodes during measurement whereas the right mastoid was included as active electrode. Vertical eye movements were registered with two electrodes positioned above and below the right eye. For measurement of horizontal eye movements two electrodes were places on the outer canthi of both eyes. Electrode impedance was kept below $10 \mathrm{k} \Omega$, with the exception of the reference and ground electrodes which were held below $5 \mathrm{k} \Omega$. EEG and EOG signals were continuously sampled at $500 \mathrm{~Hz}$ and filtered online with a bandpass filter of $0.05-120 \mathrm{~Hz}$. ERP analysis was performed using Brain Vision Analyzer software (Brain Products $\mathrm{GmbH}$, version 2.0). The continuous EEG data was first filtered offline with a low-pass filter of $30 \mathrm{~Hz}$ (24 dB/oct) (encoding-phase) or $7 \mathrm{~Hz}$ (24 dB/ oct) (maintenance-phase). Correction for horizontal eye movements and blink artifacts took place by application of the algorithm of Gratton et al. (1983), available in the Brain Vision Analyzer software package. Next, the data was epoched in windows from -200 to $9500 \mathrm{~ms}$ relative to $\mathrm{S} 1$ onset to apply baseline correction using the -200 to 0 pre-stimulus interval. In a next step separate epochs were created for encoding (0-1800 ms post-stimulus) and maintenance (5000-9500 ms post-stimulus) phases, the latter starting from the time at which the stimuli from the memory set disappeared from the screen. Trials containing EEG artifacts exceeding $\pm 75 \mu \mathrm{V}$ were rejected from the data, which was done separately for the $0-1800 \mathrm{~ms}$ epoch and the 5000-9500 ms epoch. The data was then re-referenced offline to the average of the left and right mastoids. ERP averages were computed separately for each subject in the different stimulus conditions. Only trials including correct responses to probe trials were included in the averaging procedure. For encoding, a total of 75 (range $57-102$, SD = 11.8) and 36.8 (range $24-51, S D=6.6$ ) trials remained for analyses in the semantic grouping and rehearsal condition respectively. For maintenance, 63.9 (range $31-90, \mathrm{SD}=16.6$ ) and 31.5 (range $16-47, \mathrm{SD}=8.5$ ) trials remained for analyses in the semantic grouping and rehearsal condition, respectively. Whereas the ERP analyses included more trials in the semantic grouping than rehearsal condition this could not have affected results because we used mean area amplitudes in our analyses that are not influenced by differences in signal-to-noise ratio's between conditions.

\subsection{Data analysis}

\subsubsection{Behavioral data}

Mean reaction times (RT) to correctly identified probes, mean percentage of correctly identified probes (\% hits) and mean percentage of incorrectly identified probes (\% errors) were computed in semantic grouping-picture and rehearsal-picture trials, separately for yes-response trials and no-response trials. To investigate whether memory performance was better in semantic grouping vs. rehearse trials as was hypothesized, we compared accuracy and correctresponse RTs between semantic grouping and rehearse trials in which subjects had to decide whether the probe picture (S2) was or was not part of the previously presented memory set (S1). Correct responses on yes-response trials and no-response trials are hereafter respectively referred to as hits and correct rejections. Since S1 and S2 stimuli and task demands during the retrieval phase were comparable between both conditions any differences have to be attributed to applying distinct memory strategies. A repeated measures $2 \times 2$ ANOVA was performed including the within subject-factors Trial type (hits and correct rejections) and Strategy instruction (semantic grouping-picture and rehearsal-picture). In case of a significant interaction between these two factors, paired t-tests were performed separately for hits and correct rejections. Separate analyses were done on the reaction time and accuracy data. The two-tailed alpha level for all comparisons was set at $p<.05$.

\subsubsection{ERP data}

The BESA Statistics software package was used for analyzing the ERP data (BESA GmbH, Graefelfing, Germany). In BESA statistics, information from all electrodes and all time-points is entered and non-parametric permutation testing is used to determine where in time and at which electrode-clusters differences between conditions are statistically significant (for a more elaborate description of the methods see Maris and Oostenveld, 2007; Ernst, 2004). The major advantage of this approach is that no a priori selection of electrodes or time windows is required and that only those effects are considered significant that survive correction for multiple comparisons (thereby decreasing the risk of false positive findings). The procedure that BESA Statistics follows consists of two main steps. In the first step the program performs, in our case, dependent t-tests (Student's t-test; Hays, 1988) to identify statistically significant differences between conditions for every space/time point. The condition differences resulting from this are ordered in the so-called data clusters. Then, it is determined if these initial data clusters survive permutation testing. To obtain a reliable correction for false positives, 10.000 permutations were performed. The reported data clusters survived a statistical threshold, corrected for multiple comparisons, of $p<.05$. The cluster value represents the sum of all t-values of all data points in the respective cluster.

We contrasted semantic grouping with rehearsal trials in BESA Statistics to extract strategy specific spatiotemporal activation. We chose not to compute contrasts with control conditions since, despite instructions, inspection of the grand-average ERPs and topographical maps revealed late ERP modulations possibly not due to only passively viewing the pictures. Therefore activity in control conditions was only used to control for potential confounding effects of differences in S1 stimulus displays on ERPs during the encoding interval in the two strategy conditions (see 3.3). We only report those components identified by BESA Statistics that have in earlier studies been linked to semantic grouping operations (Blanchet et al., 2007; Kiefer, 2001, 2005). For encoding we entered the entire window in BESA Statistics (0$1800 \mathrm{~ms}$ ). With respect to maintenance we were only interested in ERP activity reflecting maintenance of representations held in memory when the sensory input was not present anymore; for that reason only data following S1 offset (from 5000 to $9500 \mathrm{~ms}$ ) was entered in BESA Statistics.

\section{Results}

\subsection{Behavioral data}

Mean RTs, percentage correct responses and errors in the semantic grouping-picture and rehearsal-picture conditions (separately for hits and correct rejections) are presented in Table 1 . The $2 \times 2$ repeated measures ANOVA for the reaction time data revealed a significant interaction between Trial type and Strategy instruction, $F(1,18)=12.9$, $p<.01$. Further testing showed that while RTs to hits did not significantly differ between semantic grouping-picture and rehearse-picture

Table 1

Mean reaction times, mean percentage of correctly identified trials (accuracy in \%) and mean percentage of incorrectly identified trials (\% errors) in semantic grouping-picture trials and rehearsal-picture trials (separately for hits and correct rejections). Standard deviations are presented in parentheses.

\begin{tabular}{llll}
\hline Probe type & Reaction time (ms) & Accuracy (\%) & Errors (\%) \\
\hline Hits & & & \\
$\begin{array}{l}\text { Semantic grouping-picture } \\
\text { Rehearsal-picture }\end{array}$ & $977.3(143.3)$ & $88.9(9.4)$ & $4.3(6.3)$ \\
Correct rejections & $959.8(119.6)$ & $91.4(6.4)$ & $4.9(3.9)$ \\
Semantic grouping-picture & $930.9(135)$ & & \\
Rehearsal-picture & $963.9(135.4)$ & $87.9(13.1)$ & $3.7(5.1)$ \\
\hline
\end{tabular}


conditions, $t(18)=-1.56, p=.14$, RTs to correct rejections were significantly faster in the semantic grouping-picture than rehearse-picture condition, $t(18)=2.73, p<.05$. The $2 \times 2$ repeated measures ANOVA on the accuracy data showed that there was no main or interaction effect ( $p s>1$ ), suggesting that accuracy rates did not differ significantly between semantic grouping and rehearsal conditions. Although not depicted in Table 1, accuracy percentages were also quite high in semantic grouping-category trials ( $90 \%)$, not differing significantly from the accuracy percentages in semantic grouping-picture trials $(p=.75$ for hits and $p=.83$ for correct rejections).

\subsection{ERP data; encoding}

BESA Statistics found several significant clusters for semantic grouping vs. rehearsal trials. As mentioned in the Introduction section, we followed a hypothesis driven approach by only focusing on those ERP components that have previously been associated with semantic grouping in order to examine if these components are also modulated by rehearsal.

\subsubsection{P200}

BESA Statistics revealed an increased P200 amplitude in a time window from 164 to $268 \mathrm{~ms}$ post-stimulus for rehearsal vs. semantic grouping trials (cluster value $=-617.993, p<.05$ ). As can be seen in Fig. 2 , this P200 effect was present mainly at right temporal-parietal electrodes.

\subsection{2. $N 400$}

An increased N400 for rehearsal vs. semantic grouping conditions was found over frontal-central electrodes in a time window from 328 to 440 ms post-stimulus (cluster value $=2140.71, p<.01$; see Fig. 2 ).

\subsubsection{Late Positive Component 1 (LPC1)}

From 626 to 660 ms post-stimulus, the semantic grouping condition gave rise to an increased LPC compared to the rehearsal condition over frontal-central electrodes (cluster value $=303.343, p<.05$ ) also see Fig. 2.

\subsubsection{Late Positive Component 2 (LPC2)}

A second positivity was found from 820 to $904 \mathrm{~ms}$ post-stimulus of which the amplitude was higher in response to rehearsal compared to semantic grouping trials. As can been seen in Fig. 2, this effect was not only distributed mainly over bilateral prefrontal electrodes, but also covered some parietal electrodes (cluster value $=-1464.98, p<.01$ ).

3.3. Control for possible confounding effects of stimulus display (S1) differences between semantic grouping and rehearsal conditions

It should be noted that besides the strategy instruction manipulations the two experimental conditions differed with respect to the encoding stimulus displays. In the semantic grouping condition the four pictures belonged to two different semantic categories while in the rehearsal condition the four pictures were from four different

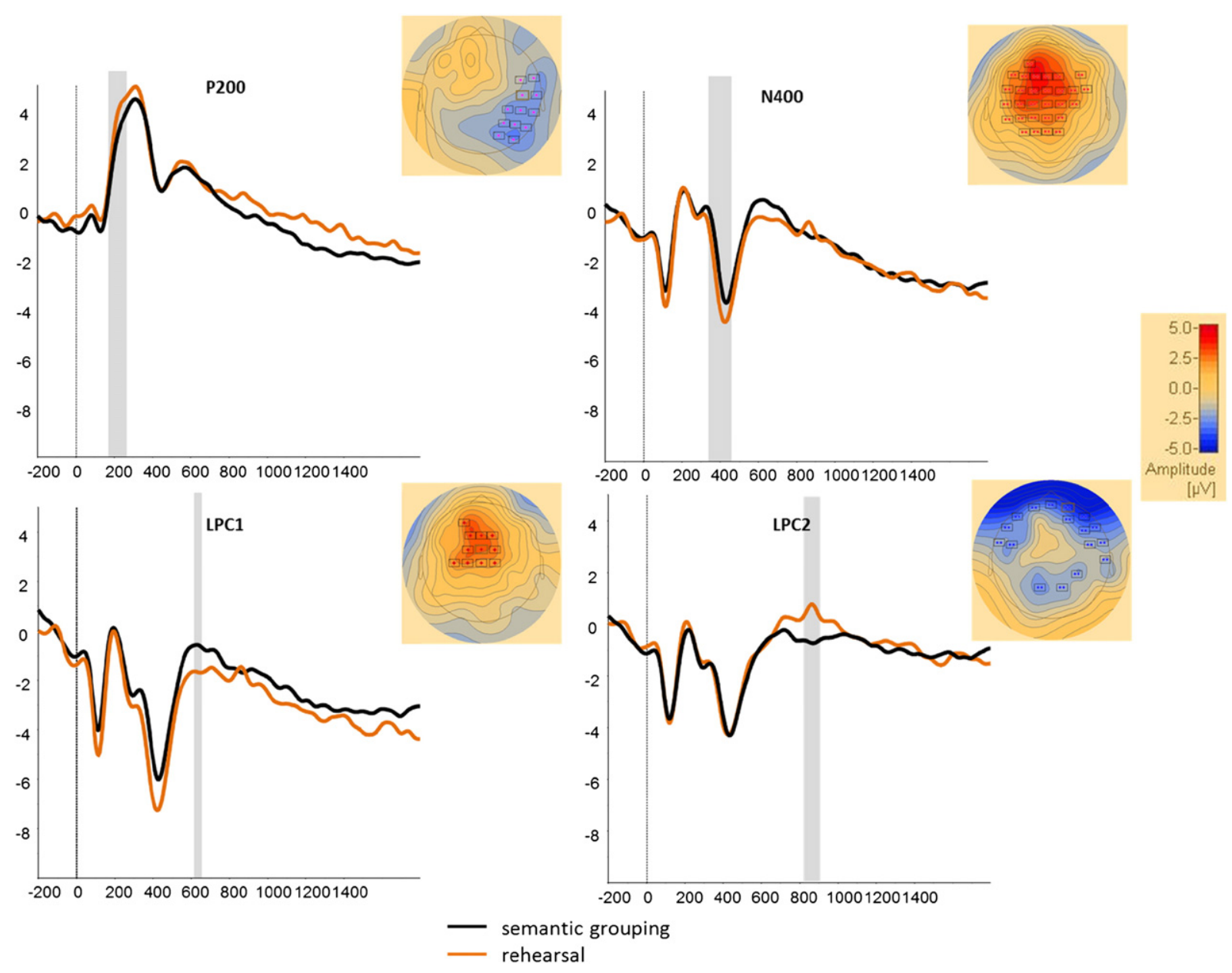

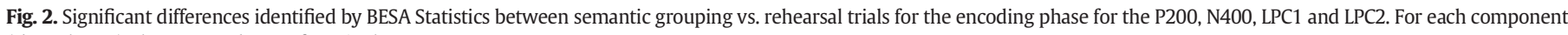
(data-cluster), the averaged wave form is shown. 
semantic categories. We performed several additional analyses to check if, and to what extent, stimulus display differences between the two experimental conditions might account for the above reported strategy effects.

As a first step, we entered the two control conditions (encoding: 01800 ) into BESA Statistics to see if in the same time windows and at the same electrodes at which the BESA strategy effects were found, any significant data clusters were found when comparing these two control conditions. No significant data clusters were found that overlapped with the reported strategy effects (i.e. on P200, N400, LPC1 and LPC2), suggesting that these effects were not due to such stimulus display differences. Secondly, as an extra check, we quantified the difference between the two control conditions, using the same electrodes/time windows as reported for the strategy effect clusters in the paper. This difference score, possibly reflecting stimulus display differences, was entered as a covariate in the semantic grouping vs. rehearsal contrast analyses (note that this was done in SPSS). This revealed that for the P200, N400 and the second late positivity (LPC2), the covariate did not substantially alter the statistics of the main condition effects (all effects remained significant), indicating that these effects were not significantly affected by stimulus display differences and are thus more likely due to strategy instruction differences. For the LPC1, however, we found that when the covariate was included, the initial significant main condition effect became trend-significant $(p=.078)$.

\subsection{ERP data; maintenance}

BESA Statistics identified three significant data-clusters in the maintenance interval. These three clusters all represented increased negative slow waves for semantically grouped versus rehearsed information, but in different time windows and at (partly) different electrodes. In a time window from 5000 to $5232 \mathrm{~ms}$ post-stimulus this effect was distributed over left frontal and left parietal electrodes (cluster value $=-2049.4$, $p<.05$ ). In a longer, overlapping time window (from 5000 to $6046 \mathrm{~ms}$ post-stimulus) this slow wave effect covered right-frontal-temporal electrodes (cluster value $=-7540.34, p<.01$ ). Finally, somewhat later in time, between 5446 and 7664 ms post-stimulus the increased slow wave for semantically grouped vs. rehearsed-trials was observed at left frontal sites (cluster value $=-8470.56, p<.01$ ) (see Fig. 3).

\section{Discussion}

Performing elaborative encoding operations on incoming stimuli, such as semantic grouping, increases the likelihood that material can be learned or later remembered. In the current study we investigated whether ERP components that were earlier shown to be modulated by semantic grouping are indeed specific to semantic grouping, or also (partly) reflect the involvement of simple rehearsal processes that rely on overlapping brain regions as those supporting elaborative encoding

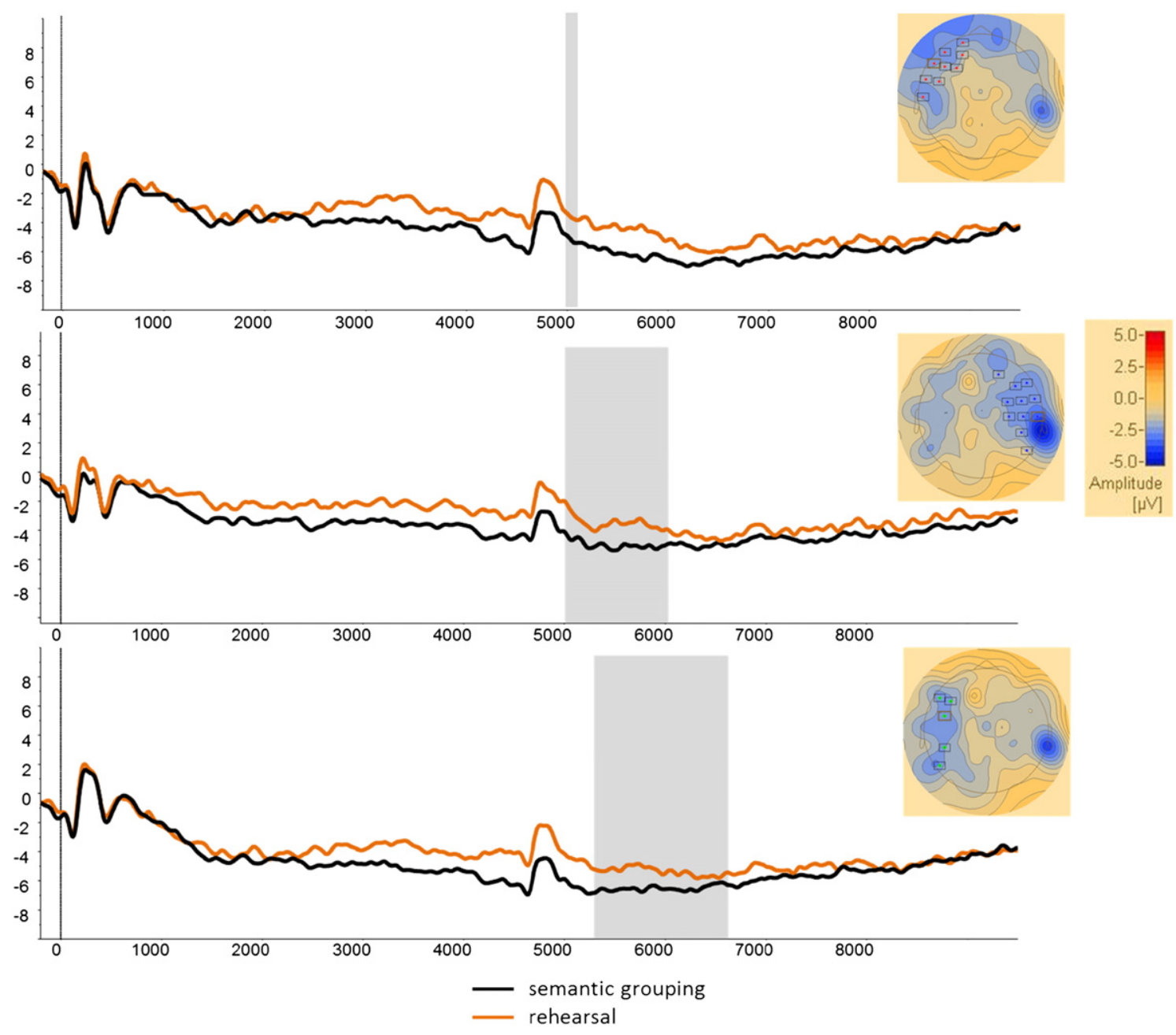

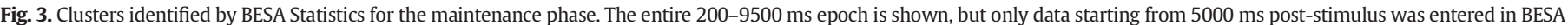

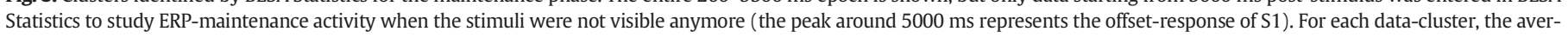
aged wave form is shown. 
(Smith and Jonides, 1999). To this purpose, during encoding and maintenance phases of a memory task, memory performance and ERP components were compared between trials in which subjects either received explicit semantic grouping or rehearse instructions.

\subsection{Behavioral results}

Accuracy was quite high ( 90\%) and did not differ between semantic grouping and rehearsal conditions. Furthermore, subjects responded faster in semantic grouping vs. rehearsal trials, but only when they had to decide that the probe stimulus was not part of the memory set (correct rejections). There was however no difference in RT for hits (correct responses on yes-trials). The faster RTs for correct rejections comply with general findings that encoding material on the basis of semantic features usually leads to better memory performance as opposed to when material is processed superficially during encoding as is the case when simple rehearsal strategies are used (Craik and Lockhart, 1972). Thus, whereas there was a benefit of semantic grouping in terms of faster recognition, this was only present on half of the (correct-rejection) trials and there were no benefits on accuracy. In the present task these modest benefits of semantic grouping on recognition performance are however not surprising since demands on working memory were deliberately kept low to be able to study ERP effects specific to semantic grouping and rehearsal without contaminating effects of load. Benefits of elaborative strategies on memory performance are known to be strongest when the to-be-stored material exceeds maximum working memory capacity. It could be argued that because the same set of pictures was shown repeatedly, priming effects could have played a role. Yet, because picture presentation was completely randomized both within and between subjects and the trial-interval was very long because of the memory requirements, we expected this to cancel out any possible priming effects. In the following paragraph the ERP results will be discussed.

\subsection{ERP results: encoding}

The earliest component that showed strategy-effects was the P200, which was increased for rehearsal versus semantic grouping trials over right temporal regions. In a prior study of Blanchet et al. the P200 was found to be increased in a condition requiring subjects to use the semantic grouping strategy (i.e. guided condition). Several factors make it however difficult to reconcile this finding with the current data. First, in the study of Blanchet et al. the P200 was enhanced in guided vs. spontaneous vs. unrelated conditions, but only in the former condition subjects received explicit strategy instructions while in this study specific instructions were given in both experimental conditions. Second, the P200 effect observed in the above study had a typical fronto-central distribution (reflecting attention-orienting activity), in contrast to the right temporal P200 topography found in the current study. Mecklinger and Muller (1996) reported a similar right temporal P200 effect that had larger amplitude in conditions that required subjects to encode object stimuli as opposed to conditions in which subjects had to encode spatial stimuli. Further, Moscovitch et al. (1995) showed by means of PET that activation in right temporal areas increased when object information was retrieved from LTM (in contrast to retrieving spatial information from LTM). Based on these findings relating right temporal brain activation to object identification processes, we tentatively suggest that the enhanced P200 in rehearsal vs. semantic grouping conditions might represent more detailed extraction of object identity information in the former, possibly because in these trials all four individual objects were analyzed. In semantic grouping trials, in contrast, the pictures themselves might have been analyzed less thoroughly because they would already undergo more elaborate processing at a higher level.

The P200 effect was followed by a modulation of the N400 component that also was larger in rehearsal compared to semantic grouping trials. This effect is quite surprising in light of studies relating an N400 to retrieval of object categories from LTM (Kiefer, 2001, 2005). Although speculative, the increased N400 in rehearsal vs. semantic grouping trials in the present study could reflect the use of additional strategies besides rehearsal in the former type of trials. Subjects might have attempted to learn the unrelated pictures in an elaborative way by for example forming mental images of them. Such an interpretation would be in line with findings by Kirchhoff and Buckner (2006) who reported that visual (imagery) strategies are among the preferred strategies to remember visually presented object pictures. In addition, a similar N400 effect has been reported in conditions where subjects used visual imagery processes (Nittono et al., 2002; Riby and Orme, 2013). Another possible explanation for the higher $\mathrm{N} 400$ to rehearse trials might be that semantic incongruity in rehearsal trials might have been higher because the encoding stimulus set consisted of object pictures from 4 different semantic categories in contrast to 4 object pictures from 2 semantic categories in the semantic grouping condition. However, entering the amplitude difference between the two control conditions (that had exactly similar related and unrelated stimulus displays but required only picture viewing) as a covariate in the semantic grouping vs. rehearsal contrasts showed that strategy-effects remained significant for the P200, N400 and LPC2 after controlling for stimulus display differences, making this explanation unlikely.

During later phases of the encoding interval, our ERP results did support those reported earlier by Blanchet et al. We also found an enhanced LPC (LPC1 in results) in semantic grouping vs. rehearsal trials between 626 and 660 ms post-stimulus, albeit at partly distinct electrodes as the LPC observed in Blanchet's study. This LPC has earlier been associated with the processing of semantic relations between items, such as deciding whether they belong to the same semantic category (Kiefer, 2001, 2005; Blanchet et al, 2007), which was in the this study only required in semantic grouping trials. Furthermore, other studies reported similarly enhanced amplitudes of a Late Positive Component during encoding especially on trials that were later successfully remembered (DM effect) and this effect (at either frontal or midline sites) was increased when items were encoded on the basis of semantic associations instead of on physical features of the stimuli (Paller et al., 1987; Weyerts et al., 1997). On this basis the enhanced LPC amplitude in the semantic grouping condition might index richer elaboration of the to-be-learned material due to retrieval of category associations. However, some critical notes need to be made. First, it has to be noted that whereas strategy effects on the N400 and LPC are reversed in terms of activation (higher activity for rehearsal for N400 and for semantic grouping for LPC), the topography of both strategy effects is comparable leaving unclear whether these are really distinct components. Second, the duration of the LPC-effect was rather short (34 ms), and also this effect became trend-significant when controlling for differences in the number of semantic categories from which the four stimuli were derived in the stimulus sets of both strategy conditions.

Finally, against expectations, from 820 to $904 \mathrm{~ms}$ post-stimulus, a second late positive cluster was found (LPC2), indicating enhanced positivity in rehearsal compared to semantic grouping trials over mainly bilateral frontal regions. Such increased slow waves have repeatedly been reported in situations in which items are associatively linked to each other in some way (Blanchet et al., 2007; Weyerts et al., 1997). During elaborative encoding WM-load is typically reduced because material is stored in more compact units (Bor et al., 2003). It may therefore be that in the current study WM-load was higher (i.e. more single items were kept in memory) in rehearsal vs. semantic grouping trials, because in the former all four individual objects were maintained while in the latter the pictures were organized into two semantic categories. Future studies are needed to investigate this.

In sum, regarding the ERP-encoding data, the present study extends prior ( fMRI) studies by demonstrating the temporal fashion in which certain strategy-related processes occur. It seems that already after $200 \mathrm{~ms}$ the first detailed analyses of pictures are made, shortly followed 
by visual imagery processes at $400 \mathrm{~ms}$ (at least in the rehearsal condition). Processes related to semantically grouping the pictures or indexing WM-load related processes are seen around 600 and $800 \mathrm{~ms}$ post-stimulus, respectively. Such temporal information could be of value in future studies including children, because it give us more information about the processing stages in which strategy-effects are possibly delayed or still immature in children or other groups or individuals that have problems with spontaneous initiation of elaborative strategies. This information might eventually contribute to cognitive theories about memory strategy development, by describing which specific cognitive processes at the time of encoding still need to develop in nonstrategic children.

\subsection{ERP results: maintenance}

The hypothesis of increased negative slow wave components in semantic grouping vs. rehearsal trials was confirmed by the current data. Such an effect was present at left frontal-parietal (from 5000 to $5232 \mathrm{~ms}$ post-stimulus), right frontal-temporal (from 5000 to $6046 \mathrm{~ms}$ post-stimulus) and left frontal (from 5446 to 7664 ms post-stimulus) electrodes. Similar increased negative slow waves over frontal regions have repeatedly been found in studies in which participants performed effortful cognitive operations upon to-be-remembered information during maintenance (Kiss et al., 2007; Ruchkin et al., 1992). In light of this literature it is suggested that elaborative rehearsal, which involves rehearsing the pictures grouped in their respective semantic categories (which was required in the semantic grouping condition), is more dependent on the available WM-resources and activation of the prefrontal cortex than simple rote rehearsal. This interpretation is supported by studies demonstrating that when implementing other types of elaborative encoding strategies, such as chunking material into smaller units or forming mental images of interacting object pairs, activation in prefrontal regions increases (Bor et al., 2004; Bor et al., 2003; Bor and Owen, 2007; Kirchhoff and Buckner, 2006). The present results add to these prior results by studying which effects are specific to semantic grouping (and are not modulated by rehearsal) and show that enhanced prefrontal cortex activation during maintenance of organized material (in this study pictures grouped on semantic category) is specific for elaborative rehearsal strategies. Further, while prior studies mainly used fMRI to localize the brain regions underlying elaborative processing (Bor et al., 2004; Bor et al., 2003; Bor and Owen, 2007; Kirchhoff and Buckner, 2006), the present study adds to the literature by providing information about the timing of such elaborative maintenance processes, arising approximately around $1500-2000 \mathrm{~ms}$ post-stimulus and lasting throughout the entire maintenance interval. Further, there was a more or less similar onset of the slow waves in both strategy conditions, suggesting that the application of the different maintenance strategies (e.g. rote or elaborative rehearsal for the maintenance of respectively unrelated and related information) started at about the same time. As mentioned, possible differences in ERP slow wave between the two experimental conditions were tested after S1 disappeared (i.e. to reflect active strategy use), and immediately from this point on the increased negative grouping-slow wave differed significantly from the rehearsalslow wave. Towards the end of the retention interval, the difference between these slow wave effects became smaller, possibly because subjects were awaiting the probe and prepared for memory retrieval.

Although we did not perform source analysis on our data, the increased activation during maintenance of grouped (vs. ungrouped) material above mainly left and right frontal regions is in accord with human lesion and neuroimaging studies showing that the PFC plays an essential role in maintaining information in memory in an organized way (Gershberg and Shimamura, 1995; Prabhakaran et al., 2000; Stuss et al., 1994; Wendelken et al., 2008). The current slow waves mainly observed above left and right frontal regions might index maintenance of distinct types of material. The activation observed above left frontal areas is also consistent with prior ERP studies showing that slow waves above left frontal regions arise when keeping phonological material in WM (Ruchkin et al., 1992; Bosch et al., 2001), suggesting that the present left frontal slow waves index maintenance of the names of the S1-pictures/categories. We cannot answer the question which specific processes evoked the left and right frontal activation, but some speculations can be made based on results of prior studies. Increased left frontal activation, mostly in Inferior Frontal Gyrus (IFG) or Dorso-lateral Prefrontal cortex (DLPFC), has in several studies been linked specifically to the processing of semantic features or relations between stimuli during encoding, as opposed to for instance processing of physical relations (Demb et al., 1995; Petersen et al., 1988; Hawco et al., 2013; Innocenti et al., 2010; Savage et al., 2001). Although the current left frontal cluster was found during maintenance of information grouped on semantic category, it might index IFG or DLPFC activity evoked by the processing/updating of semantic relations between items during maintenance.

Regarding the right frontal clusters, in light of studies reporting modulations of ERP slow waves above right frontal areas in response to objectmaintenance processes (Mecklinger and Pfeifer, 1996; Mecklinger, 1998), the right frontal slow waves possibly reflects retention of object information. The right frontal cortex has been suggested to play a role in the successful application of semantic grouping strategies (Savage et al., 2001). Further, neuroimaging research has demonstrated that activation in the right prefrontal cortex predicts later memory (Brewer et al., 1998; Henson et al., 1999; Kirchhoff et al., 2000; Wagner et al., 1998). Although speculatively, the enhanced right prefrontal cortex activation in related (vs. unrelated) trials in the maintenance phase of the task in the current study could reflect the efficient implementation of elaborative rehearsal strategies. Although the above mentioned studies investigated activity during encoding, because of the limited time resolution of neuroimaging methods these effects might actually (also) represent maintenancerelated processes.

Finally, the increased negative slow waves were also distributed above several (left and right) parietal-temporal sites during elaborative vs. rote rehearsal. Previous ERP work on slow wave-WM relations demonstrated that maintaining in WM spatial information elicits slow waves above posterior brain regions (Mecklinger and Meinshausen, 1998). On this basis we suggest that subjects might have also remembered the spatial location in which the pictures were presented in the $2 \times 2$ matrix, which possibly aided memory. Various neuroimaging studies have associated activation of parietal-temporal brain regions with memory storage (Coull et al., 1996; Paulesu et al., 1993; Pochon et al., 2001) and the current posterior strategy-effects might hence be indicative of such memory storage processes. Taken together, in agreement with prior studies the present data seem to indicate that a network of prefrontal-parietal brain regions is involved during maintenance of information that is organized or structured (e.g. storage in categories or chunks).

\subsection{Conclusion}

To the best of our knowledge this is the first ERP study that investigated to what extent the ERP-effects related earlier to semantic grouping are indeed specific to this strategy, or are also modulated by the use of rehearsal. We indeed found that especially during relatively early phases of the encoding interval several ERP components previously linked to grouping material on semantic category during encoding (P200, N400 and late sustained wave) were in fact strongly influenced by processes related to rehearsal in our picture memory task. The fronto-central encoding-LPC and maintenance-related fronto-temporal-parietal ERP slow waves were modulated in the expected direction, i.e. were specifically sensitive to the semantic grouping instruction (and not to rehearsal). The present study highlights the importance of carefully controlling and manipulating the type of WM-strategies used (versus the use of noinstruction control conditions) when examining the neural correlates underlying elaborative encoding strategies. 


\section{Acknowledgments}

The authors are grateful to Andre Opstal for programming the experiment and to Marjolein Spronk and Roefje Janssen for their help in data collection.

\section{References}

Baddeley, 2000. The episodic buffer: a new component of working memory? Trends Cogn. Sci. 4, 417-423.

Bjorklund, D.F., de Marchena, M.R., 1984. Developmental shifts in the basis of organization in memory: the role of associative versus categorical relatedness in children's free recall. Child Dev. 55, 952-962.

Blanchet, S., Gagnon, G., Bastien, C., 2007. Event-related potential study of dynamic neural mechanisms of semantic organizational strategies in verbal learning. Brain Res. 1170, 59-70.

Bor, D., Owen, A.M., 2007. A common prefrontal-parietal network for mnemonic and mathematical recoding strategies within working memory. Cereb. Cortex 17, 778-786.

Bor, D., Duncan, J., Wiseman, R.J., Owen, A.M., 2003. Encoding strategies dissociate prefrontal activity from working memory demand. Neuron 37, 361-367.

Bor, D., Cumming, N., Scott, C.E., Owen, A.M., 2004. Prefrontal cortical involvement in verbal encoding strategies. Eur. J. Neurosci. 19, 3365-3370.

Bosch, V., Mecklinger, A., Friederici, A.D., 2001. Slow cortical potentials during retention of object, spatial, and verbal information. Cogn. Brain Res. 10, 219-237.

Brewer, J.B., Zhao, Z., Desmond, J.E., Glover, G.H., Gabrieli, J.D., 1998. Making memories: brain activity that predicts how well visual experience will be remembered. Science 281, 1185-1187.

Coull, J.T., Frith, C.D., Frackowiak, R.S., Grasby, P.M., 1996. A fronto-parietal network for rapid visual information processing: a PET study of sustained attention and working memory. Neuropsychologia 34, 1085-1095.

Courchesne, E., Hillyard, S.A., Galambos, R., 1975. Stimulus novelty, task relevance and the visual evoked potential in man. Electroencephalogr. Clin. Neurophysiol. 39, 131-143.

Cowan, N., 2001. The magical number 4 in short-term memory: a reconsideration of mental storage capacity. Behav. Brain Sci. 24, 87-114 (discussion 114-185).

Craik, F.I.M., Lockhart, R.S., 1972. Levels of processing: a framework for memory research. J. Verbal Learn. Verbal Behav. 11, 671-684.

Cycowicz, Y.M., Friedman, D., Rothstein, M., Snodgrass, J.G., 1997. Picture naming by young children: norms for name agreement, familiarity, and visual complexity. J. Exp. Child Psychol. 65, 171-237.

Demb, J.B., Desmond, J.E., Wagner, A.D., Vaidya, C.J., Glover, G.H., Gabrieli, J.D., 1995. Semantic encoding and retrieval in the left inferior prefrontal cortex: a functional MRI study of task difficulty and process specificity. J. Neurosci. 15, 5870-5878.

Egeland, J., Nordby Johansen, S., Ueland, T., 2010. Do low-effort learning strategies mediate impaired memory in ADHD? J. Learn. Disabil. 43, 430-440.

Ernst, M.D., 2004. Permutation methods: a basis for exact inference. Stat. Sci. 19, 676-685

Fletcher, P.C., Shallice, T., Dolan, R.J., 1998. The functional roles of prefrontal cortex in episodic memory. Encoding. Brain 121, 1239-1248.

Gershberg, F.B., Shimamura, A.P., 1995. Impaired use of organizational strategies in free recall following frontal lobe damage. Neuropsychologia 33, 1305-1333.

Gratton, G., Coles, M.G.H., Donchin, E., 1983. A new method for offline removel of ocular artifact. Electroencephalography and clinical neurophysiology 55, 468-484.

Hawco, C., Armony, J.L., Lepage, M., 2013. Neural activity related to self-initiating elaborative semantic encoding in associative memory. Neuroimage 67, 273-282.

Hays, W.L., 1988. Statistics, 4th ed. Holt, Rinehart \& Winston Inc., Fort Worth, TX

Henson, R.N., Rugg, M.D., Shallice, T., Josephs, O., Dolan, R.J., 1999. Recollection and familiarity in recognition memory: an event-related functional magnetic resonance imaging study. J. Neurosci. 19, 3962-3972.

Hillyard, S.A., Picton, T.W., 1978. On and off components in the auditory evoked potential. Percept. Psychophys. 24, 391-398.

Innocenti, I., Giovannelli, F., Cincotta, M., Feurra, M., Polizzotto, N.R., Bianco, G., et al., 2010. Event-related rTMS at encoding affects differently deep and shallow memory traces. Neuroimage 53, 325-330.

Kiefer, M., 2001. Perceptual and semantic sources of category-specific effects: eventrelated potentials during picture and word categorization. Mem. Cogn. 29, 100-116.

Kiefer, M., 2005. Repetition-priming modulates category-related effects on event-related potentials: further evidence for multiple cortical semantic systems. J. Cogn. Neurosci. 17, 199-211.

Kirchhoff, B.A., Buckner, R.L., 2006. Functional-anatomic correlates of individual differences in memory. Neuron 51, 263-274.

Kirchhoff, B.A., Wagner, A.D., Maril, A., Stern, C.E., 2000. Prefrontal-temporal circuitry for episodic encoding and subsequent memory. J. Neurosci. 20,6173-6180.

Kirchhoff, B.A., Anderson, B.A., Barch, D.M., Jacoby, L.L., 2012. Cognitive and neural effects of semantic encoding strategy training in older adults. Cereb. Cortex 22, 788-799.

Kiss, I., Watter, S., Heisz, J.J., Shedden, J.M., 2007. Control processes in verbal working memory: an event-related potential study. Brain Res. 1172, 67-81.
Kutas, M., Hillyard, S.A., 1980. Reading senseless sentences: brain potentials reflect semantic incongruity. Science 207, 203-205.

Mandler, G., 1967. Organization and memory. In: Spence, K.W., Spence, J.T. (Eds.), The Psychology of Learning and Motivation vol. 1. Academic Press, New York, pp. 327-372.

Mangels, J.A., Picton, T.W., Craik, F.I., 2001. Attention and successful episodic encoding: an event-related potential study. Cogn. Brain Res. 11, 77-95.

Maris, E., Oostenveld, R., 2007. Nonparametric statistical testing of EEG- and MEG-data. J. Neurosci. Methods 164, 177-190.

Mecklinger, A., 1998. On the modularity of recognition memory for object form and spatial location: a topographic ERP analysis. Neuropsychologia 36, 441-460.

Mecklinger, A., Muller, N., 1996. Dissociations in the processing of "what" and "where" information in working memory: an event-related potential analysis. J. Cogn. Neurosci. 8, 453-473.

Mecklinger, A., Pfeifer, E., 1996. Event-related potentials reveal topographical and temporal distinct neuronal activation patterns for spatial and object working memory. Cogn. Brain Res. 4, 211-224.

Mecklinger, A., Meinshausen, R., 1998. Recognition memory for object forms and spatial locations: An event-related potential study. Memory and Cognition 26, 1068-1088.

Miller, G.A., 1956. The magical number seven plus or minus two: some limits on our capacity for processing information. Psychol. Rev. 63, 81-97.

Miotto, E.C., Savage, C.R., Evans, J.J., Wilson, B.A., Martins, M.G., Iaki, S., et al., 2006. Bilatera activation of the prefrontal cortex after strategic semantic cognitive training. Hum. Brain Mapp. 27, 288-295.

Moscovitch, C., Kapur, S., Kohler, S., Houle, S., 1995. Distinct neural correlates of visua long-term memory for spatial location and object identity: a positron emission tomography study in humans. Proc. Natl. Acad. Sci. U. S. A. 92, 3721-3725.

Nittono, H., Suehiro, M., Hori, T., 2002. Word imageability and N400 in an incidental memory paradigm. Int. J. Psychophysiol. 44, 219-229.

Paller, K.A., Kutas, M., Mayes, A.R., 1987. Neural correlates of encoding in an incidental learning paradigm. Electroencephalogr. Clin. Neurophysiol. 67, 360-371.

Paulesu, E., Frith, C.D., Frackowiak, R.S., 1993. The neural correlates of the verbal component of working memory. Nature 362, 342-345.

Petersen, S.E., Fox, P.T., Posner, M.I., Mintun, M., Raichle, M.E., 1988. Positron emission tomographic studies of the cortical anatomy of single-word processing. Nature 331 $585-589$.

Picton, T.W., Hillyard, S.A., 1974. Human auditory evoked potentials. II. Effects of attention. Electroencephalogr. Clin. Neurophysiol. 36, 191-199.

Pochon, J.B., Levy, R., Poline, J.B., Crozier, S., Lehericy, S., Pillon, B., et al., 2001. The role of dorsolateral prefrontal cortex in the preparation of forthcoming actions: an fMRI study. Cereb. Cortex 11, 260-266.

Prabhakaran, V., Narayanan, K., Zhao, Z., Gabrieli, J.D., 2000. Integration of diverse information in working memory within the frontal lobe. Nat. Neurosci. 3, 85-90.

Riby, L.M., Orme, E., 2013. A familiar pattern? Semantic memory contributes to the enhancement of visuo-spatial memories. Brain Cogn. 81, 215-222.

Ruchkin, D.S., Johnson, R., Grafman, J., Canoune, H., Ritter, W., 1992. Distinctions and similarities among working-memory processes - an event-related potential study. Cogn. Brain Res. 1, 53-66.

Rypma, B., Berger, J.S., D'Esposito, M., 2002. The influence of working-memory demand and subject performance on prefrontal cortical activity. J. Cogn. Neurosci. 14, 721-731.

Savage, C.R., Deckersbach, T., Heckers, S., Wagner, A.D., Schacter, D.L., Alpert, N.M., et al. 2001. Prefrontal regions supporting spontaneous and directed application of verbal learning strategies: evidence from PET. Brain 124, 219-231.

Schleepen, T.M.J., Jonkman, L.M., 2012. Children's use of semantic organizational strategies is mediated by working memory capacity. Cogn. Dev. 27, 255-269.

Schneider, W., Kron, V., Hunnerkopf, M., Krajewski, K., 2004. The development of young children's memory strategies: First findings from the Wurzburg Longitudinal Memory Study. Journal of Experimental Child Psychology 88, 193-209.

Smith, E.E., Jonides, J., 1999. Storage and executive processes in the frontal lobes. Science 283, 1657-1661.

Stuss, D.T., Alexander, M.P., Palumbo, C.L., Buckle, L., Sayer, L., Poque, J., 1994. Organizational strategies of patients with unilateral or bilateral frontal lobe injury in word list learning tasks. Neuropsychologia 8, 355-373.

Wagner, A.D., Schacter, D.L., Rotte, M., Koutstaal, W., Maril, A., Dale, A.M., et al., 1998 Building memories: remembering and forgetting of verbal experiences as predicted by brain activity. Science $281,1188-1191$

Wegesin, D.J., Jacobs, D.M., Zubin, N.R., Ventura, P.R., Stern, Y., 2000. Source memory and encoding strategy in normal aging. J. Clin. Exp. Neuropsychol. 4, 455-464.

Wendelken, C., Bunge, S.A., Carter, C.S., 2008. Maintaining structured information: an investigation into functions of parietal and lateral prefrontal cortices. Neuropsychologia 46, 665-678.

Weyerts, H., Tendolkar, I., Smid, H.G., Heinze, H.J., 1997. ERPs to encoding and recognition in two different inter-item association tasks. Neuroreport 8, 1583-1588. 